\title{
Discovery of methyl silane and confirmation of silyl cyanide in IRC +10216
}

\author{
J. Cernicharo ${ }^{1}$, M. Agúndez ${ }^{1}$, L. Velilla Prieto ${ }^{1}$, M. Guélin ${ }^{2}$, J. R. Pardo ${ }^{1}$, C. Kahane ${ }^{3}$, C. Marka ${ }^{4}$, C. Kramer ${ }^{4}$, \\ S. Navarro ${ }^{4}$, G. Quintana-Lacaci ${ }^{1}$, J. P. Fonfría ${ }^{1}$, N. Marcelino ${ }^{1}$, B. Tercero ${ }^{1}$, E. Moreno ${ }^{1}$, S. Massalkhi ${ }^{1}$, \\ M. Santander-García ${ }^{1}$, M. C. McCarthy ${ }^{5}$, C. A. Gottlieb ${ }^{5}$, and J. L. Alonso ${ }^{6}$ \\ ${ }^{1}$ Group of Molecular Astrophysics, ICMM, CSIC, C/ Sor Juana Inés de La Cruz 3, 28049 Madrid, Spain \\ e-mail: jose.cernicharo@csic.es \\ 2 Institut de Radioastronomie Millimétrique, 300 rue de la Piscine, 38406 St-Martin d'Hères, France \\ 3 Université Grenoble Alpes, CNRS, IPAG, 38000 Grenoble, France \\ ${ }^{4}$ Instituto de Radioastronomía Milimétrica, Av. Divina Pastora 7, Local 20, 18012 Granada, Spain \\ 5 Harvard-Smithsonian Center for Astrophysics, Cambridge, MA 02138, and School of Engineering \& Applied Sciences, \\ Harvard University, Cambridge, MA 02138, USA \\ ${ }^{6}$ Grupo de Espectroscopía Molecular (GEM), Edificio Quifima, Área de Química-Física, Laboratorios de Espectroscopía y \\ Bioespectroscopía, Parque Científico UVa, Unidad Asociada CSIC, Universidad de Valladolid, 47011 Valladolid, Spain
}

Received 29 July 2017 / Accepted 4 September 2017

\section{ABSTRACT}

\begin{abstract}
We report the discovery in space of methyl silane, $\mathrm{CH}_{3} \mathrm{SiH}_{3}$, from observations of ten rotational transitions between 80 and $350 \mathrm{GHz}$ ( $J_{\mathrm{u}}$ from 4 to 16 ) with the IRAM $30 \mathrm{~m}$ radio telescope. The molecule was observed in the envelope of the C-star IRC +10216 . The observed profiles and our models for the expected emission of methyl silane suggest that the it is formed in the inner zones of the circumstellar envelope, 1-40 $R_{*}$, with an abundance of $(0.5-1) \times 10^{-8}$ relative to $\mathrm{H}_{2}$. We also observed several rotational transitions of silyl cyanide $\left(\mathrm{SiH}_{3} \mathrm{CN}\right)$, confirming its presence in IRC +10216 in particular, and in space in general. Our models indicate that silyl cyanide is also formed in the inner regions of the envelope, around $20 R_{*}$, with an abundance relative to $\mathrm{H}_{2}$ of $6 \times 10^{-10}$. The possible formation mechanisms of both species are discussed. We also searched for related chemical species but only upper limits could be obtained.
\end{abstract}

Key words. stars: individual: IRC+10216 - stars: carbon - astrochemistry - stars: AGB and post-AGB

\section{Introduction}

CW Leo, located $\sim 120 \mathrm{pc}$ from us, is a carbon-rich asymptotic giant branch (AGB) variable star with a period of 630-670 days and an amplitude of $\sim 1$ mag in the $K$ band (Menten et al. 2012, and references therein). Due to its close proximity, IRC +10216 , the circumstellar envelope of CW Leo, is one of the brightest infrared (IR) sources in the sky; its exceptionally rich molecular content has led several authors to study it in detail (see Cernicharo et al. 2000, and references therein). Nearly 50\% of the known interstellar species have been observed in this C-rich envelope. Many of them were discovered in space for the first time there. The silicon-carbon species $\mathrm{Si}_{n} \mathrm{C}_{m}$, which are abundant in IRC +10216 , could play an important role as gas-phase precursors of $\mathrm{SiC}$ dust grains. The simplest members of this family, $\mathrm{SiC}$ (Cernicharo et al. 1989) and $\mathrm{SiC}_{2}$ (Thaddeus et al. 1984), are known to be present in this circumstellar envelope. Recently, the first species with two $\mathrm{Si}$ atoms $\left(\mathrm{Si}_{2} \mathrm{C}\right)$ has also been discovered there (Cernicharo et al. 2015a). Other Sibearing species found in IRC $+10216, c-\mathrm{SiC}_{3}$ and $\mathrm{SiC}_{4}$, show line profiles that indicate that they are formed in the external regions of the envelope where other radicals are formed under the action of the galactic ultraviolet field (Guélin et al. 1993).

\footnotetext{
$\star$ This work was based on observations carried out with the IRAM $30-\mathrm{m}$ telescope. IRAM is supported by INSU/CNRS (France), MPG (Germany), and IGN (Spain).
}

The formation of dust grains can be simplified as a two-step process: formation of a nucleation seeds close to the AGB star, and grain growth through the addition of species containing refractory elements at high temperature and other species at lower temperature and larger distances from the star. There are, however, many unknowns in this picture of events, starting from the fundamental step of the formation of the nucleation seeds, which are essentially refractory compounds (Gail 2010). The presence of $\mathrm{SiC}$ grains in $\mathrm{C}$-rich $\mathrm{AGB}$ stars was confirmed by the detection of an emission band at $\sim 11.3 \mu \mathrm{m}$ (Treffers \& Cohen 1974), which has been found towards several C-rich evolved stars with the IRAS and ISO satellites (Yang et al. 2004). Unfortunately, the molecular precursors of $\mathrm{SiC}$ dust grains have not yet been identified. $\mathrm{SiC}$ molecules are detected in the external regions of IRC +10216 , but not in the inner zones (Cernicharo et al. 1989; Patel et al. 2013; Velilla Prieto et al. 2015). As discussed by Cernicharo et al. (2015a), among the silicon-carbon species $\mathrm{Si}_{n} \mathrm{C}_{m}, \mathrm{SiC}_{2}$, and $\mathrm{Si}_{2} \mathrm{C}$ could play an important role in the formation of $\mathrm{SiC}$ dust grains in $\mathrm{C}$-rich envelopes. These species have large abundances in the inner zones of the envelope (Cernicharo et al. 2010, 2015a; Velilla Prieto et al. 2015). Other Si-bearing species studied recently in IRC +10216 with ALMA and at mid-IR wavelengths are $\mathrm{SiS}$ and $\mathrm{SiO}$ (Velilla Prieto et al. 2015; Fonfría et al. 2015), which, together with $\mathrm{SiC}_{2}$ and $\mathrm{Si}_{2} \mathrm{C}$, account for a significant fraction of the available silicon (Agúndez et al. 2012; Velilla Prieto et al. 2015). SiCN and SiNC 
have been also detected in the external layers of the envelope by Guélin et al. (2000, 2004), but with much lower abundances than $c-\mathrm{SiC}_{3}$ and $\mathrm{SiC}_{4}$.

Other potential Si-bearing species involved in the growth of dust grains could be silane, $\mathrm{SiH}_{4}$, the first hydrogenated Si-bearing species detected in IRC +10216 and in space (Goldhaber \& Betz 1984). However, it is unclear where in the envelope this species is formed. Keady \& Ridgway (1993) suggest a formation region at $\sim 40$ stellar radii, based on the analysis of the line profiles of several ro-vibrational lines. However, Monnier et al. (2000) claims that the formation of silane occurs at $\sim 80 R_{*}$ based on interferometric observations of one of its ro-vibrational lines. The derived abundance of silane, $\sim 2 \times 10^{-7}$ relative to $\mathrm{H}_{2}$, is larger than predicted by thermochemical equilibrium by several orders of magnitude and thus it has been suggested that $\mathrm{SiH}_{4}$ could be formed by catalytic reactions at the surface of dust grains (Keady \& Ridgway 1993).

The presence of $\mathrm{SiH}_{4}$ could lead to the formation of species containing the groups $\mathrm{SiH}_{3}$ and $\mathrm{SiH}_{2}$. Recently, Agúndez et al. (2014) have presented the tentative detection of three rotational lines of silyl cyanide $\left(\mathrm{SiH}_{3} \mathrm{CN}\right)$. In this letter we present the discovery of methyl silane $\left(\mathrm{CH}_{3} \mathrm{SiH}_{3}\right)$ in IRC +10216 , based on the detection of ten rotational lines with the IRAM $30 \mathrm{~m}$ telescope. We also report the detection of six rotational lines of $\mathrm{SiH}_{3} \mathrm{CN}$, confirming the presence of silyl cyanide in this source and in space. Organosilicon molecules are widely used in terrestrial chemistry applications. The detection of the two organosilicon molecules $\mathrm{CH}_{3} \mathrm{SiH}_{3}$ and $\mathrm{SiH}_{3} \mathrm{CN}$ might help the understanding of silicon-carbon chemistries in the inner envelope of AGB stars. We have also searched for transitions of $\mathrm{CH}_{2} \mathrm{SiH}_{2}$ and $\mathrm{H}_{2} \mathrm{CSi}$, but only upper limits have been obtained.

\section{Observations and results}

Data for this work have been acquired over the last 30 years and the observations are described in detail in Appendix A. These data have revealed several hundreds of spectral lines which cannot be assigned to any known molecular species collected in the public spectral databases CDMS (Müller et al. 2005) and JPL (Pickett 1998), and in the MADEX code (Cernicharo 2012). Most of these lines show the characteristic U-shaped emission arising from the external layers, or flat-topped emission from the middle and external layers. All lines, except the narrow features arising from the dust formation zone, have linewidths of $29 \mathrm{~km} \mathrm{~s}^{-1}$ (Cernicharo et al. 2000, 2015a). At frequencies above $250 \mathrm{GHz}$ a significant number of lines are very narrow and come from the dust formation zone of IRC +10216 (see, e.g., Patel et al. 2011; Cernicharo et al. 2013, 2015a). Among the unidentified lines observed with the IRAM 30m telescope, we have been able to assign ten harmonically related lines (see Fig. 1) with profiles showing line broadening that increases with frequency (thereby eliminating the possibility of hyperfine structure as the source of line broadening). The most obvious source of this behavior is the presence of several $K$ components for a given $J \rightarrow(J-1)$ rotational transition, i.e., the carrier must be a symmetric top. The rotational quantum numbers are integers and the derived rotational constant is around $10970 \mathrm{MHz}$. A quick look through the MADEX code allows these features to be assigned to methyl silane $\left(\mathrm{CH}_{3} \mathrm{SiH}_{3}\right)$, a derivative of methane $\left(\mathrm{CH}_{4}\right)$ and silane $\left(\mathrm{SiH}_{4}\right)$, both of which are abundant species in IRC +10216 .

The rotational spectrum of $\mathrm{CH}_{3} \mathrm{SiH}_{3}$ was implemented in MADEX from a fit to the rotational lines of the ground vibrational state reported by Meerts \& Ozier (1982) and
Wong et al. (1983), which cover frequencies up to $285.1 \mathrm{GHz}$ and transitions with $J_{\max }=13$ and $K_{\max }=12$. The standard deviation of the fit is $70 \mathrm{kHz}$, and thus frequencies can be predicted with an uncertainty below $0.3 \mathrm{MHz}$ up to the transition $J=25 \rightarrow 24(K=012)$, whose frequency is around $547 \mathrm{GHz}$. The dipole moment of the molecule is $\mu=0.7346 \mathrm{D}$ (Ozier \& Meerts 1982). The $K$ ladders of each $J \rightarrow(J-1)$ rotational transition in methyl silane are more tightly spaced in frequency than those of other symmetric rotors such as $\mathrm{CH}_{3} \mathrm{CN}$. This peculiarity, together with the fact that lines in IRC +10216 have widths of $\sim 29 \mathrm{~km} \mathrm{~s}^{-1}$, results in severe blending of the $K=0-3$ components of each $J \rightarrow(J-1)$ rotational transition in the spectra in IRC+10216 (see Fig. 1).

The $J=4 \rightarrow 3,5 \rightarrow 4,8 \rightarrow 7,11 \rightarrow 10,12 \rightarrow 11,13 \rightarrow 12$, and $16 \rightarrow 15$ lines are free of blending, or only slightly blended with the features of other species, as indicated in Fig. 1. The $J=6 \rightarrow 5$ ( $K=0$ is at $131618.485 \mathrm{MHz}$ ) line is blended on its red wing with the $J=23 \rightarrow 22$ line of $\mathrm{C}^{13} \mathrm{CCS}$ at $131612.142 \mathrm{MHz}$, for which we expect an intensity of $2 \mathrm{mK}$ from the observation of the adjacent $J=22 \rightarrow 21$ and $J=24 \rightarrow 23$ lines. The $J=7 \rightarrow 6$ line is blended at its blue edge with a strong line of $\mathrm{NaCN}$ and this same species also affects the $J=10 \rightarrow 9$ line of methyl silane at its red edge. In any case, these two lines of $\mathrm{CH}_{3} \mathrm{SiH}_{3}$ can be clearly distinguished in the spectra. The $J=14 \rightarrow 13$ line is fully blended with the strong $J=17 \rightarrow 16$ emission line of SiS in its $v=1$ state. No data are available for the $J=9 \rightarrow 8$ and $J=15 \rightarrow 14$ transitions.

The similarity of the line profiles of the ten observed transitions provides very strong evidence in support of the identification of this species in IRC +10216 . Additional evidence comes from the comparison between the observed line profiles with those calculated with a non-LTE excitation radiative transfer model. The derived rotational temperatures for the low- $J$ lines are $\simeq 40-50 \mathrm{~K}$, while for higher $J$ and $K$ lines the derived values for $T_{\text {rot }}$ are 100-200 K. Non-LTE calculations were performed using MADEX (Cernicharo 2012) and are based on a multi-shell large velocity gradient (LVG) method, in which we adopted the rate excitation coefficients through inelastic collisions of $\mathrm{CH}_{3} \mathrm{CN}$ (Green 1986) and the physical structure of the envelope of Agúndez et al. (2012) with the downward revision of the density profile by Cernicharo et al. (2013). The inner radius of the $\mathrm{CH}_{3} \mathrm{SiH}_{3}$ spatial distribution was initially taken as $40 R_{*}$, the same derived for $\mathrm{SiH}_{4}$ by Keady \& Ridgway (1993). The best fit to the low $-J$ lines corresponds to an abundance of methyl silane of $5 \times 10^{-9}$ relative to $\mathrm{H}_{2}$ from 40 to $600 R_{*}$, with a vanishing abundance beyond $600 R_{*}$ due to photodissociation. This model, however, does not reproduce the high- $J$ lines correctly. A much better fit for all lines is obtained if the abundance relative to $\mathrm{H}_{2}$ is $10^{-8}$ from the photosphere to $40 R_{*}$. We note, however, that the regions inwards of $20 R_{*}$ are poorly traced by the observations and, in addition, the intensities of the high- $J$ lines could be affected by infrared pumping (methyl silane has various strong bands at mid-IR wavelengths; Randić 1962). The line profiles resulting from this latter model $\left(\mathrm{X}\left(\mathrm{CH}_{3} \mathrm{SiH}_{3}\right)=10^{-8}\right.$ for $1<r<40 R_{*}$ and $5 \times 10^{-9}$ for $40<r<600 R_{*}$ ), shown in green in Fig. 1, are in excellent agreement with the observed lines, including the high- $K$ components of high- $J$ transitions. The high abundance inferred for methyl silane, in layers inner to those of $\mathrm{SiH}_{4}$, indicates that the molecule is formed in a warm dense environment by thermochemical equilibrium, by chemical reactions involving abundant radicals, or by reactions at the surface of the grains (see below).

Silyl cyanide, $\mathrm{SiH}_{3} \mathrm{CN}$, was tentatively identified in IRC +10216 by Agúndez et al. (2014) through three rotational 
J. Cernicharo et al.: Discovery of $\mathrm{CH}_{3} \mathrm{SiH}_{3}$
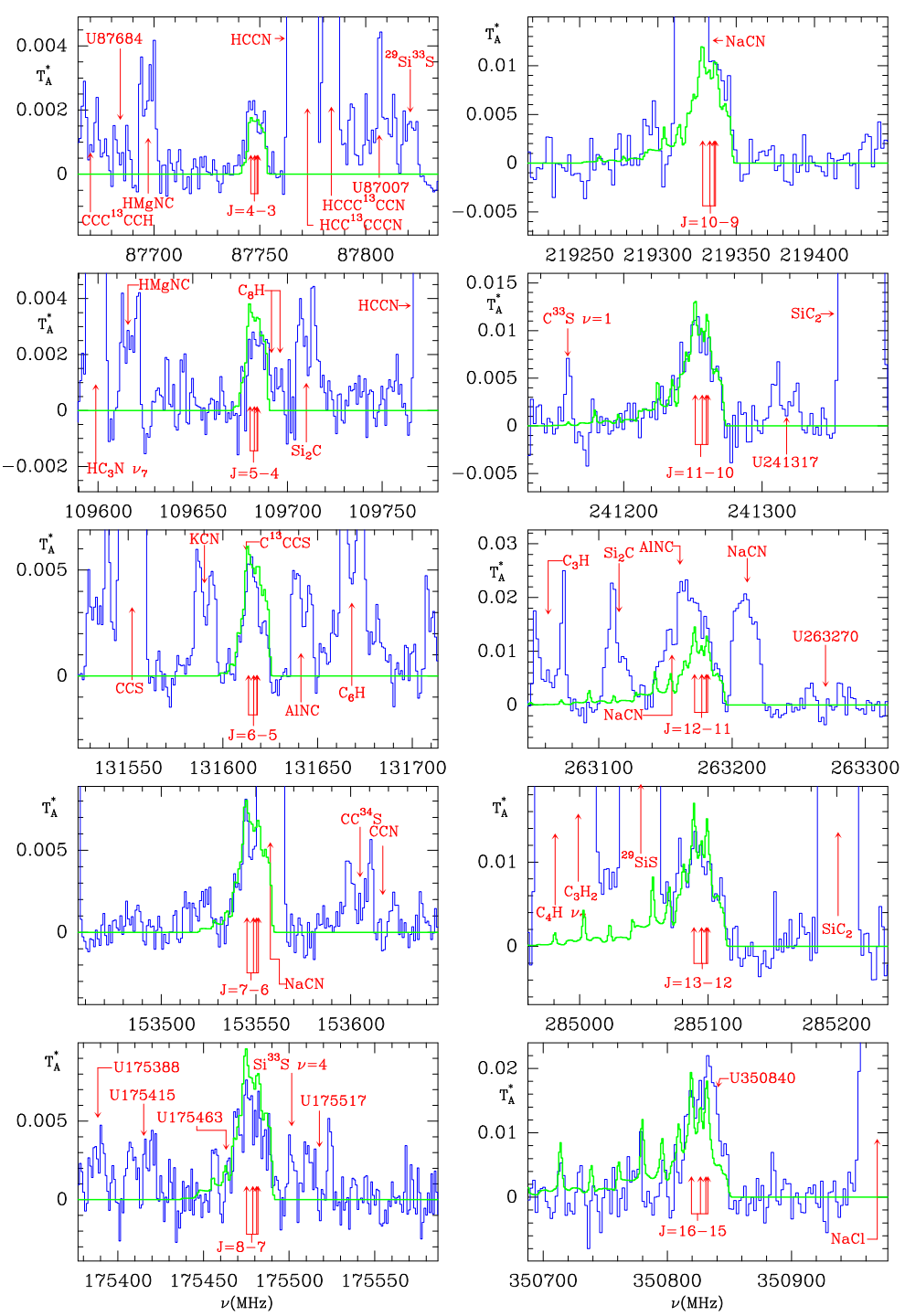

Fig. 1. Observed rotational lines of methyl silane, $\mathrm{CH}_{3} \mathrm{SiH}_{3}$, towards IRC+10216 in antenna temperature (in $K$ ). The spectral resolution is $1 \mathrm{MHz}$ for lines below $200 \mathrm{GHz}$, and $2 \mathrm{MHz}$ above that frequency. Rest frequencies are indicated for an assumed $L S R$ velocity of the source of $-26.5 \mathrm{~km} \mathrm{~s}^{-1}$. The arrows indicate the position of the $K=0,1,2$, and 3 components of each $J \rightarrow(J-1)$ rotational transition. In most cases the $K=0$ and 1 components appear superposed. The identification of other features found in the spectra is indicated. Some of the $\mathrm{CH}_{3} \mathrm{SiH} \mathrm{H}_{3}$ lines are partially blended, but their red or blue parts are still clearly visible in the spectra. Green lines correspond to the emerging line profiles calculated with the model discussed in the text.

transitions lying in the $\lambda=3 \mathrm{~mm}$ band. In Fig. A.1 we show the six rotational transitions of $\mathrm{SiH}_{3} \mathrm{CN}$ lying in spectral regions of the $\lambda=2$ and $3 \mathrm{~mm}$ bands in which we have sensitive data. The $J=8 \rightarrow 7$ line is not shown because it is blended with a line of $\mathrm{C}_{7} \mathrm{H}$. The $J=9 \rightarrow 8$ and $J=10 \rightarrow 9$ lines were reported by Agúndez et al. (2014), but since then new data have been added (see Fig. A.1) and now both lines emerge more clearly above the noise. The $J=11 \rightarrow 10$ line is not shown here because it is blended with a line of $\mathrm{C}_{3} \mathrm{~N}$ in the $v_{5}=1$ vibrational state (see spectrum in Agúndez et al. 2014). The $J=13 \rightarrow 12$ line, not shown, is fully blended with lines of $\mathrm{Si}_{2} \mathrm{C}, \mathrm{C}_{3} \mathrm{~N} v_{5}=1$, and $\mathrm{C}_{6} \mathrm{H} v_{11}=1$. The $J=14 \rightarrow 13$ through $J=17 \rightarrow 16$ lines are not blended and are clearly detected. Higher $J$ lines have expected intensities below the sensitivity of our data. Hence, for $\mathrm{SiH}_{3} \mathrm{CN}$, a total of six unblended rotational lines showing the $K$-ladder structure are detected in IRC +10216 . Consequently, our new data confirm the presence of silyl cyanide in IRC+10216. We have used a model similar to that of methyl silane and the best fit (green lines in Fig. A.1) is obtained if $\mathrm{SiH}_{3} \mathrm{CN}$ is present with an abundance relative to $\mathrm{H}_{2}$ of $6 \times 10^{-10}$ between 20 and $700 R_{*}$.
The calculated line profiles show a remarkable agreement with the observations in terms of both intensity and line shape.

\section{Discussion}

Methyl silane could be formed in the inner envelope where $\mathrm{C}_{2} \mathrm{H}_{2}, \mathrm{C}_{2} \mathrm{H}_{4}$, methane, and silane are formed. Formation under thermochemical equilibrium in the surroundings of the star is unlikely because the abundance of $\mathrm{CH}_{3} \mathrm{SiH}_{3}$ predicted with data from Allendorf \& Melius (1992), is vanishingly small within the first stellar radii of the star.

Formation of methyl silane in the outer layers could be driven by reactions involving the silyl and silylene radicals, $\mathrm{SiH}_{3}$ and $\mathrm{SiH}_{2}$, and the methyl and methylene radicals, $\mathrm{CH}_{3}$ and $\mathrm{CH}_{2}$, which could be produced by photodissociation of silane and methane, respectively. The radicals $\mathrm{SiH}_{n}$ and $\mathrm{CH}_{n}$ (with $n=2-3$ ) react slowly with $\mathrm{H}_{2}$, and hence these species are potential precursors of $\mathrm{CH}_{3} \mathrm{SiH}_{3}$. The kinetics of the reaction between $\mathrm{SiH}_{3}$ and $\mathrm{CH}_{4}$ have not been studied, but it is endothermic by $64 \mathrm{~kJ} \mathrm{~mol}^{-1}$ in the channel yielding $\mathrm{CH}_{3} \mathrm{SiH}_{3}+\mathrm{H}$. The reaction of $\mathrm{CH}_{3}$ with $\mathrm{SiH}_{4}$ has a barrier of $\sim 3500 \mathrm{~K}$ to produce $\mathrm{CH}_{4}$ 
and $\mathrm{SiH}_{3}$ by $\mathrm{H}$ abstraction (Arthur \& Bell 1978). This reaction is nearly thermoneutral in the formation of $\mathrm{CH}_{3} \mathrm{SiH}_{3}$, although it is not known whether this channel has a substantial energy barrier. Including this reaction in a chemical model of IRC +10216 (Agúndez et al. 2017) with a rate constant of $10^{-10} \mathrm{~cm}^{3} \mathrm{~s}^{-1}$ results in a $\mathrm{CH}_{3} \mathrm{SiH}_{3}$ abundance of a few $10^{-10}$ relative to $\mathrm{H}_{2}$, i.e., lower than that observed by more than one order of magnitude.

Methyl silane has been observed in a laboratory cold plasma of $\mathrm{SiH}_{4} / \mathrm{CH}_{4} / \mathrm{He}$ by Catherine et al. (1981), who suggested that the three-body association reactions $\mathrm{SiH}_{2}+\mathrm{CH}_{4}, \mathrm{SiH}_{3}+\mathrm{CH}_{3}$, and $\mathrm{CH}_{2}+\mathrm{SiH}_{4}$ are the main pathways to $\mathrm{CH}_{3} \mathrm{SiH}_{3}$ in their experiment. These reactions, however, are spin forbidden and thus would need to have an intersystem crossing to proceed as radiative associations. In any case, even if we adopt a very optimistic rate of $10^{-12} \mathrm{~cm}^{3} \mathrm{~s}^{-1}$ for these three reactions, the chemical model predicts that methyl silane is produced with an abundance of only $\sim 10^{-11}$ relative to $\mathrm{H}_{2}$.

Agúndez et al. (2014) have suggested that $\mathrm{SiH}_{3} \mathrm{CN}$ could be formed by a route similar to that of $\mathrm{CH}_{3} \mathrm{CN}$, i.e., involving the precursor ion $\mathrm{SiH}_{3} \mathrm{CNH}^{+}$instead of $\mathrm{CH}_{3} \mathrm{CNH}^{+}$. A similar way to produce methyl silane could involve the ion $\mathrm{CH}_{3} \mathrm{SiH}_{4}^{+}$. However, more recent ALMA observations, which put the inner radius of $\mathrm{CH}_{3} \mathrm{CN}$ in IRC +10216 at only $1-2^{\prime \prime}$, probably rule out these ionic pathways (Agúndez et al. 2015). An alternative formation mechanism for methyl silane and silyl cyanide are catalytic reactions on the surface of dust grains by hydrogenation of silicon-carbon species. We could expect significant abundances of $\mathrm{Si}_{n} \mathrm{C}_{m}, \mathrm{C}_{n}$, and $\mathrm{Si}$-bearing species on grain surfaces in C-rich envelopes, although their relative abundances and their potential to be hydrogenated are poorly known.

The detection of $\mathrm{CH}_{3} \mathrm{SiH}_{3}$ and $\mathrm{SiH}_{3} \mathrm{CN}$ suggests that other Si-bearing species could be present in the envelope. For example, the reaction of $\mathrm{C}\left({ }^{3} \Pi\right)$ with $\mathrm{SiH}_{4}$ produces $\mathrm{SiCH}_{3}$ and $\mathrm{SiCH}_{2}$ without an entrance barrier (Sakai et al. 1989; Lu et al. 2008). However, the analogous reaction of $\mathrm{Si}$ with $\mathrm{CH}_{4}$ could have a large activation barrier (Sakai et al. 1989). $\mathrm{SiCH}_{2}$ has been observed in the laboratory and searched for towards IRC +10216 by Izuha et al. (1996). They derived an upper limit to the column density of $5.8 \times 10^{13} \mathrm{~cm}^{-2}$. With our new data this upper limit is reduced by a factor of 3 . This molecule has a very low dipole moment, $\sim 0.3 \mathrm{D}$ (Izuha et al. 1996), which makes it difficult to detect. The $\mathrm{H}_{2} \mathrm{SiC}$ isomer has a larger dipole moment $(2.7 \mathrm{D})$, but it lies $\sim 88 \mathrm{Kcal} \mathrm{mol}^{-1}$ above $\mathrm{H}_{2} \mathrm{CSi}$. Another species that could be produced in reactions of $\mathrm{SiH}_{n}$ and $\mathrm{CH}_{n}$ is $\mathrm{H}_{2} \mathrm{CSiH}_{2}$. Its rotational spectrum has been measured by Bailleux et al. (1997) and it has a dipole moment of 0.66 D (Maroulis 2011). We searched for it and derive an upper limit to its column density of $4 \times 10^{12} \mathrm{~cm}^{-2}$. $\mathrm{SiH}_{3}$ itself has some rotational lines in the submillimeter domain, but the rotational frequencies can only be roughly estimated from the infrared observations of Yamada \& Hirota (1986). We searched for ro-vibrational lines of the $\mathrm{SiH}_{3} v_{2}$ band in the mid-IR data $\left(720-820 \mathrm{~cm}^{-1}\right)$ published by Fonfría et al. (2008) and found no significant absorption features at the positions of the strongest lines where the rms noise of the data was $0.2 \%$ of the continuum level.

Note added in proof. The rotational spectrum of $\mathrm{SiH}_{3} \mathrm{NC}$, an isomer of silyl cyanide, was recently measured in the laboratory by K. L. K. Lee, M. C. McCarthy, and C. A. Gottlieb. Details will be presented elsewhere, and an astronomical search is underway.

Acknowledgements. We acknowledge funding support from Spanish MINECO under grants AYA2012-32032, AYA2016-75066-C2-1-P, and CSD2009-00038, and from the European Research Council under grant ERC-2013-SyG 610256 (NANOCOSMOS).

\section{References}

Agúndez, M., Fonfría, J. P., Cernicharo, J., et al. 2012, A\&A, 543, A48 Agúndez, M., Cernicharo, J., \& Guélin, M. 2014, A\&A, 570, A45 Agúndez, M., Cernicharo, J., Quintana-Lacaci, G., et al. 2015, ApJ, 814, 143 Agúndez, M., Cernicharo, J., Quintana-Lacaci, G., et al. 2017, A\&A, 601, A4 Allendorf, M. D., \& Melius, C. F. 1992, J. Phys. Chem., 96, 428 Arthur, N. L., \& Bell, T. N. 1978, Rev. Chem. Intermed., 2, 37 Bailleux, S., Bogey, M., Demaison, J., et al. 1997, J. Chem. Phys., 106, 10016 Catherine, Y., Turban, G., \& Grolleau, B. 1981, Thin Solid Films, 76, 23 Cernicharo, J. 1985, Internal IRAM report (Granada: IRAM)

Cernicharo, J. 2012, in ECLA-2011: Proc. of the European Conference on Laboratory Astrophysics, eds. C. Stehl, C. Joblin, \& L. d'Hendecourt (Cambridge: Cambridge Univ. Press), EAS Pub. Ser., 251

Cernicharo, J., Gottlieb, C. A., Guélin, et al. 1989, ApJ, 341, L25

Cernicharo, J., Guélin, M., \& Kahane, C. 2000, A\&AS, 142, 181

Cernicharo, J., Guélin, M., Agúndez, M., et al. 2008, ApJ, 688, L83

Cernicharo, J., Waters, L. B. F. M., Decin, L., et al. 2010, A\&A, 521, L8

Cernicharo, J., Daniel, F., Castro-Carrizo, A., et al. 2013, ApJ, 778, L25

Cernicharo, J., Teyssier, D., Quintana-Lacaci, G., et al. 2014, ApJ, 796, L21

Cernicharo, J., McCarthy, M. C., Gottlieb, C. A., et al. 2015a, ApJ, 806, L3

Cernicharo, J., Marcelino, N., Agúndez, M., \& Guélin, M. 2015b, A\&A, 575, A91

Fonfría, J. P., Agúndez, M., Tercero, B., et al. 2006, ApJ, 646, L127

Fonfría, J. P., Cernicharo, J., Richter, M., et al. 2008, ApJ, 673, 445

Fonfría, J. P., Cernicharo, J., Richter, M., et al. 2015, MNRAS, 453, 439

Gail, H. P., 2010, Lect. Notes Phys., 815, 61

Goldhaber, D. M., \& Betz, A. L. 1984, ApJ, 279, L55

Green, S. 1986, ApJ, 309, 331

Guélin, M., Lucas, R., \& Cernicharo, J. 1993, A\&A, 280, L19

Guélin, S., Muller, S., Cernicharo, J., et al. 2000, A\&A, 363, L9

Guélin, S., Muller, S., Cernicharo, J., et al. 2004, A\&A, 426, L49

Izuha, M., Yamamoto, S., \& Saito, S. 1996, J. Chem. Phys., 105, 4923

Keady, J. J., \& Ridgway, S. T. 1993, ApJ, 406, 199

Lu, I.-C., Chen, W.-K., Huang, W.-J., \& Lee, S.-H. 2008, J. Chem. Phys., 129, 164304

Maroulis, G. 2011, Chem. Phys. Lett., 505, 5

Menten, K. M., Reid, M. J., Kaminski, T., \& Claussen, M. J. 2012, A\&A, 543, A73

Meerts, W. L., \& Ozier, I. 1982, J. Mol. Spectr., 94, 38

Monnier, J. D., Danchi, W. C., Hale, D. S., et al. 2000, ApJ, 543, 868

Müller, H. S. P., Schlöder, F., Stutzki, J., \& Winnewisser, G. 2005, J. Mol. Struct., 742,215

Pardo, J. R., Cernicharo, J., \& Serabyn, E. 2001, IEEE Trans. Antennas and Propagation, 49, 12

Patel, N. A., Young, K. H., Gottlieb, C. A., et al. 2011, ApJS, 193, 17

Patel, N. A., Gottlieb, C. A., \& Young, K. H. 2013, ApJS, 193, 17

Ozier, I., \& Meerts, W. L. 1982, J. Mol. Spectr., 93, 164

Pickett, H. M., Poynter, R. L., Cohen, E. A., et al. 1998, J. Quant. Spectr. Rad. Transf., 60, 883

Quintana-Lacaci, G., Agúndez, M., Cernicharo, J., et al. 2016, A\&A, 592, A51

Randić, M. 1962, Spectrochim. Acta, 18, 115

Sakai, S., Deisz, J., \& Gordon, M. S. 1989, J. Phys. Chem., 93, 1888

Thaddeus, P., Cummins, S. E., \& Linke, R. A. 1984, ApJ, 283, L45

Treffers, R., \& Cohen M. 1974, ApJ, 188, 545

Velilla Prieto L., Cernicharo, J., Quintana-Lacaci, G., et al. 2015, ApJ, 805, L13

Wong, M., Ozier, I., \& Meerts, W. L. 1983, J. Mol. Spectr., 102, 89

Yamada, C., \& Hirota, E. 1988, Phys. Rev. Lett., 56, 923

Yang, X., Chen, P., \& He, J. 2004, A\&A, 414, 1049 


\section{Appendix A: Observations}

In the course of searches for new molecules in IRC +10216 we have covered a large fraction of the $\lambda=3,2,1$, and $0.8 \mathrm{~mm}$ spectrum with the IRAM $30 \mathrm{~m}$ telescope (see Cernicharo et al. 2015a). In the $\lambda=3 \mathrm{~mm}$ window, the data acquired over the last 30 years cover the $70-116 \mathrm{GHz}$ frequency range with a very low noise level ( $T_{\mathrm{A}}^{*} \mathrm{rms}$ is $0.6-1.5 \mathrm{mK}$ per $1 \mathrm{MHz}$ channel). Some of these data have been published by Cernicharo et al. (2008, 2015a) and Agúndez et al. (2014). The oldest $\lambda=$ $2 \mathrm{~mm}$ data come from the line survey of IRC +10216 carried out by Cernicharo et al. (2000), which have been complemented with additional observations obtained during the search for specific molecular species (see, e.g., Guélin et al. 2000, 2004; Fonfría et al. 2006; Agúndez et al. 2012; Cernicharo et al. 2015a) and from a series of new sensitive observations performed in January and April 2017. The $\lambda=2 \mathrm{~mm}$ merged data are also very sensitive $\left(T_{\mathrm{A}}^{*} \mathrm{rms}\right.$ is $0.6-1.3 \mathrm{mK}$ per $1 \mathrm{MHz}$ channel). The $\lambda=1$ and $0.8 \mathrm{~mm}$ data were acquired from a variety of observations, including the searches cited above, a monitoring study of IRC +10216 to search for time variability that was begun in 2015, a line survey in the $290-355 \mathrm{GHz}$ frequency range with the EMIR receivers in 2010 and 2011, and dedicated sensitive observations to observe lines of methyl silane in January 2017.

The observations carried out before 2010 used a filter bank or autocorrelator providing a spectral resolution of $1 \mathrm{MHz}$ at frequencies below $280 \mathrm{GHz}$, while at higher frequencies we used two autocorrelators with $2 \mathrm{MHz}$ of spectral resolution and 4 $\mathrm{GHz}$ bandwidth. The most recent observations used the new fast Fourier transform spectrometers, which cover a bandwidth of $2 \times 16 \mathrm{GHz}$ with a spectral resolution of $\sim 200 \mathrm{kHz}$. In all observations the secondary mirror was wobbled by $\pm 90^{\prime \prime}$ at a rate of $0.5 \mathrm{~Hz}$, which ensures flat baselines when combined with the dry weather conditions (sky opacity at $225 \mathrm{GHz}$ was below 0.1 in most observations). The system noise temperature $T_{\text {sys }}$ was 100-400 K (depending on the frequency).

The selected observing method, with the off position located at $180^{\prime \prime}$ from the star, provides reference data free from emission for all molecular species because their spatial extent is restricted to a region $\leq 20-30^{\prime \prime}$ from the star (see, e.g., Guélin et al. 1993; Agúndez et al. 2015, 2017; Velilla Prieto et al. 2015; Quintana-Lacaci et al. 2016). Only CO has a larger spatial extent (see Cernicharo et al. 2015b). The antenna temperature $T_{\mathrm{A}}^{*}$, was corrected for atmospheric absorption using the ATM package (Cernicharo 1985; Pardo et al. 2001). The main beam antenna temperature can be obtained by dividing $T_{\mathrm{A}}^{*}$ by the main beam efficiency of the telescope ${ }^{1}$. Calibration uncertainties during such a large observing period have been adopted to be $10 \%, 15 \%, 20 \%$, and $30 \%$ at $\lambda=3,2,1$, and $0.8 \mathrm{~mm}$, respectively. Additional uncertainties could arise from the fluctuations of line intensities with time induced by the variation of the stellar infrared flux, an effect that has been recently discovered by Cernicharo et al. (2014). All data were analyzed using the GILDAS package ${ }^{2}$.

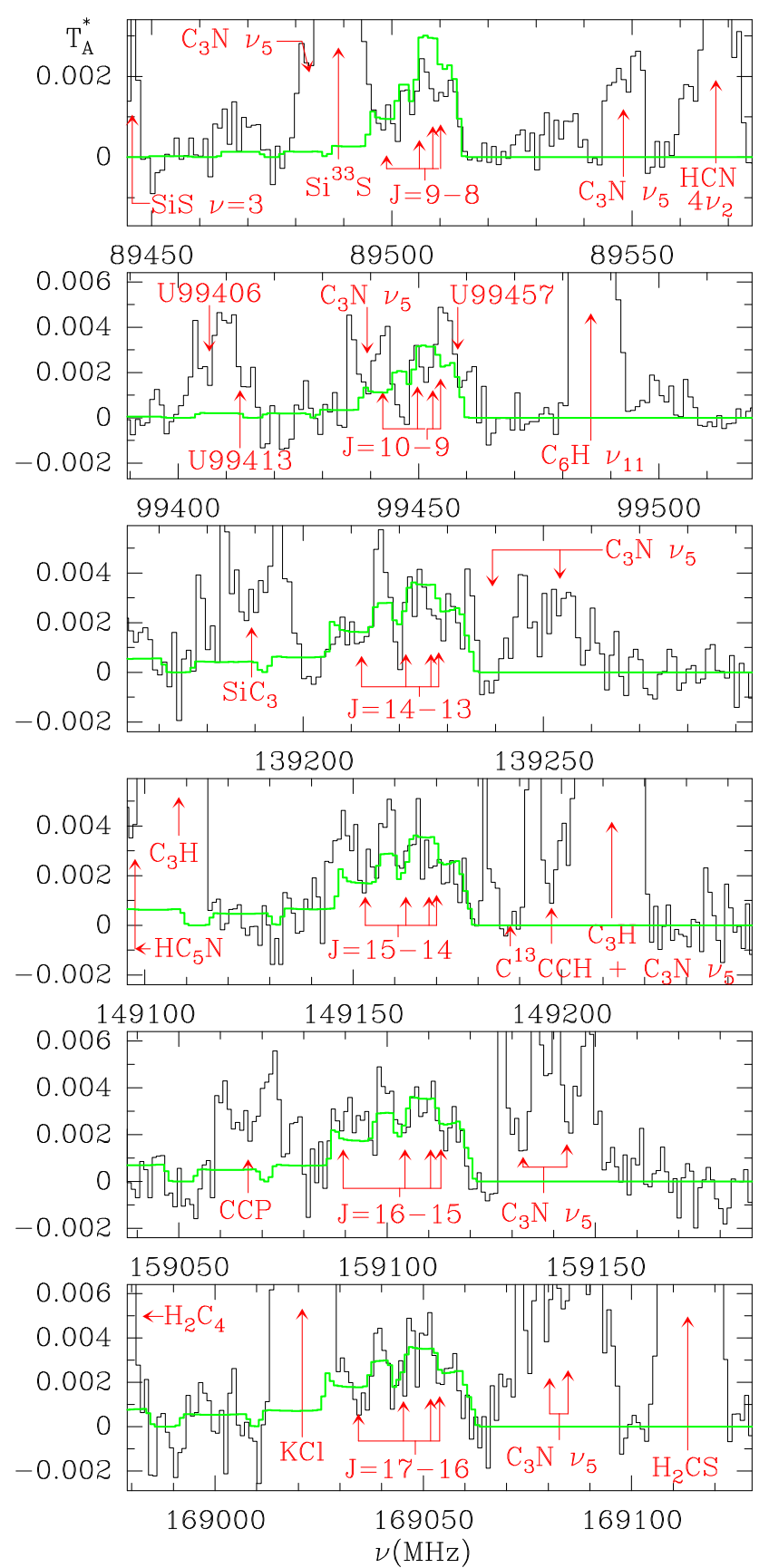

Fig. A.1. Observed rotational lines of silyl cyanide, $\mathrm{SiH}_{3} \mathrm{CN}$, toward IRC +10216 . Spectral resolution is $1 \mathrm{MHz}$ and the intensity scale is antenna temperature. Rest frequencies are indicated for an assumed $L S R$ velocity of the source of $-26.5 \mathrm{~km} \mathrm{~s}^{-1}$. The arrows indicate the position of the $K=0,1,2$, and 3 components of each $J \rightarrow(J-1)$ rotational transition. The identification of other features found in the spectra is indicated. Green lines correspond to the emerging line profiles calculated with the model discussed in the text.

\footnotetext{
1 http://WWW.iram.es/IRAMES/mainWiki/ Iram30mEfficiencies

2 http://WWW.iram. fr/IRAMFR/GILDAS
} 